\title{
Changes in induced regulatory T cells by interleukin 35 during atopic dermatitis* $^{*}$
}

\author{
Soheil Tavakolpour ${ }^{1}$
}

DOI: http://dx.doi.org/10.1590/abd1806-4841.20165504

It was with great interest that I read the recently published, brief communication by Roesner et al., which analyzed the circulation frequency of forkhead box P3 (Foxp3)+ regulatory T cells (Tregs) in adult patients with atopic dermatitis (AD). ${ }^{1}$ The study found a positive correlation between these cells and disease severity. Recently, several researchers have focused on the relatively newly identified Tregs, which are induced by interleukin (IL)-35 and suppress effector $\mathrm{T}$ cell function. This type of Treg was primarily described by Collison et al. in 2010 and termed iTr35. ${ }^{2}$ Unfortunately, despite the critical roles of $i \operatorname{Tr} 35$ in regulating immune responses, its role in different autoimmune diseases like AD remains largely unknown. It was reported that $i \operatorname{Tr} 35$ cells are phenotypically and functionally distinct from previously identified Tregs, including Foxp ${ }^{+}$and Tr1 Tregs. These cells mediate suppression of effector T cells in an IL10 and TGF- $\beta$ independent manner. Additionally, it was shown that iTr35 can suppress T helper (Th) 2 cell differentiation, which benefits AD patients suffering from Th2 dominancy. Furthermore, iTr35 produces IL-35 in an autocrine manner. Indeed, through the promotion of iTr35, a positive feedback loop of IL-35 production and iTr35 development could be established. Because the functions of autoantibody-producing B cells are strongly dependent on T cells, it seems that suppression of autoreactive $\mathrm{T}$ cell functions, especially Th2 cells via iTr35, may impair autoreactive B cell production. Although this type of cell plays a critical role in AD theoretically, no study

Received on 13.12.2015

Approved by the Advisory Board and accepted for publication on 20.02.2016 * Work performed at the Infectious Diseases and Tropical Medicine Research Center, Shahid Beheshti University of Medical Sciences - Tehran, Iran.

Financial Support: None.

Conflict of Interest: None.

1 Skin Research Center, Shahid Beheshti University of Medical Sciences Tehran, Iran.

C2016 by Anais Brasileiros de Dermatologia

\section{REFERENCES}

1. Roesner LM, Floess S, Witte T, Olek S, Huehn J, Werfel T. Foxp3+ regulatory T cells are expanded in severe atopic dermatitis patients. Allergy. 2015;70:1656-60.

2. Collison LW, Chaturvedi V, Henderson AL, Giacomin PR, Guy C, Bankoti J, et al. IL-35-mediated induction of a potent regulatory $T$ cell population. Nat Immunol. 2010;11:1093-101

3. Shen P, Roch T, Lampropoulou V, O'Connor RA, Stervbo U, Hilgenberg E, et al. IL-35-producing $B$ cells are critical regulators of immunity during autoimmune and infectious diseases. Nature. 2014;507:366-70.

4. Wang RX, Yu CR, Dambuza IM, Mahdi RM, Dolinska MB, Sergeev YV, et al. Interleukin-35 induces regulatory B cells that suppress autoimmune disease. Nat Med. 2014;20:633-41. measured frequency of iTr35 in patients with this disease. Given the crucial role of IL-35 in differentiating these cells, it is suggested that determining IL-35 levels could also assist in addressing the question of how iTr35 Tregs change during AD. Understanding this issue will help to provide a new therapeutic approach toward the inhibition of aggressive responses from immune systems. It seems that this new subset of Tregs may decrease during AD. However, further studies are needed to confirm this hypothesis. Recently, it was revealed that IL-35 can also induce a new subset of regulatory B cells (Bregs). ${ }^{3}$ These newly emerged Bregs may be a critical factor in various autoimmune diseases, including $\mathrm{AD} .{ }^{4}$ By analyzing the populations of these Bregs, in addition to their functions, a new biological treatment based on inducing the cells may be initiated. In $\mathrm{AD}$ patients, it can be speculated that IL- $35^{+}$Bregs may decrease and that dysfunction may be observed. If so, induction of IL-35 could be used to remit severe AD patients. In our recent studies, we examined the increased IL-4 levels in pemphigus patients and the inhibition of this cytokine with a newly emerged drug for moderate to severe $\mathrm{AD}$; dupilumab was suggested. 5,6 For future studies, we recommend measuring iTr35 and IL- $35^{+}$Bregs as well as IL-35 levels in AD patients. This could help to establish a new biological treatment for AD patients.]
MAILING ADDRESS:

Soheil Tavakolpour

Infectious Diseases and Tropical Medicine Research Center Shahid Beheshti University of Medical Sciences

Tabnak Street, Shahid Chamran Highway

Evin, Tehran 19395-4719

Iran

E-mail: soheil.tavakolpour@gmail.com

5. Tavakolpour S, Tavakolpour V. Interleukin 4 inhibition as a potential therapeutic in pemphigus. Cytokine. 2016 Jan; $77: 189-95$.

6. Tavakolpour S. Dupilumab: a revolutionary emerging drug in atopic dermatitis and its possible role in pemphigus. Dermatologic therapy. 2016;29:299.

How to cite this article: Tavakolpour S. Changes in induced regulatory T cells by interleukin 35 during atopic dermatitis. An Bras Dermatol. 2016;91(5):703. 